\title{
Interpretation of a heterogeneous radiological response as tumor heterogeneity or a non-tumor diagnosis: A case report
}

\author{
JEONG-SEON RYU ${ }^{1,2}$, LUCIA KIM ${ }^{3}$, WOO CHUL KIM ${ }^{4}$, JUNG-SOO KIM ${ }^{1,2}$,

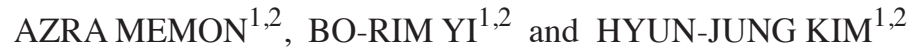 \\ ${ }^{1}$ Center for Lung Cancer; Departments of ${ }^{2}$ Internal Medicine, ${ }^{3}$ Pathology and ${ }^{4}$ Radiation Oncology, \\ Inha University Hospital, Incheon 400-711, Republic of Korea
}

Received October 30, 2014; Accepted August 17, 2015

DOI: $10.3892 / \mathrm{ol} .2015 .3709$

\begin{abstract}
Heterogeneous radiological responses (HRRs) among tumor lesions are usually observed following chemotherapy or radiation treatment in cancer patients. When HRR is observed after chemotherapy or radiation treatment, a change in anticancer treatment is recommended due to the clinically high suspicion of resistance in the majority of cases. The present study reports the case report of a patient with limited-stage small cell lung cancer, diagnosed by bronchoscopy, who received concurrent chemoradiation therapy. Upon response evaluation, the majority of lesions irradiated had nearly completely disappeared following treatment, but one lesion had apparently increased in size. For histological confirmation, a percutaneous needle biopsy for the lesion was performed, however, non-specific necrosis was found and the results were inconclusive for the differentiation of other causes from tumor necrosis. Several acid-fast bacilli were identified on Ziehl-Neelsen staining for the differential diagnosis. This case suggests that a non-tumor diagnosis should be considered when HRR presents after treatment that is expected to result in a higher response rate, particularly in tuberculosis endemic areas.
\end{abstract}

\section{Introduction}

Lung cancer is the leading cause of cancer-associated mortality worldwide and small-cell lung cancer (SCLC) accounts for $\sim 15 \%$ of all primary lung cancers (1). Notably, SCLC and non-small cell lung cancer (NSCLC) differ in terms of tumor biology, clinical presentation and response to treatment. Mediastinal or hilar lymphadenopathies, which are associated with a cough and dyspnea, are more common on clinical presentation of SCLC than NSCLC (2). Platinum doublet therapy with

Correspondence to: Professor Jeong-Seon Ryu, Center for Lung Cancer, Inha University Hospital, 27 Inhang-Ro, Jung-Gu, Incheon 400-711, Republic of Korea

E-mail: jsryu@inha.ac.kr

Key words: small cell lung cancer, tuberculosis, tumor heterogeneity etoposide or irinotecan is the standard treatment for SCLC and $>60 \%$ of SCLC patients respond to treatment $(3,4)$. The five-year survival rate of SCLC patients is <5\% (4). Recently, advances in high-throughput technologies, such as next generation sequencing, have led to the identification of a number of mutations, including RICTOR, KIT and PIK3CA, which are considered to exert a crucial role in SCLC tumor biology (5). Treatment response is evaluated in order to determine the therapeutic effect and to modify the treatment regimen when progressive disease is observed in a variety of cancers. Heterogeneous radiological response (HRR) among tumor lesions, where certain lesions decrease in size or disappear and other lesions increase in size, is usually observed following chemotherapy or radiation treatment in cancer patients and is estimated to occur in $15-36 \%$ of cancer cases $(6,7)$. With advances in high-throughput technologies, such as next-generation sequencing, intra- or inter-tumoral genomic heterogeneity has been highlighted in the area of molecular oncology and can improve our understanding of this response (8). For example, inter-tumoral heterogeneity of an EGFR mutation has been well described in adenocarcinoma (9). Therefore, the HRR leads clinicians to switch to another anticancer regimen due to the clinically high suspicion of resistance, as histological confirmation is not feasible in the majority of cases. However, when the response is presented, a non-tumor diagnosis should be considered where its relevant treatment is required. The present study reports the case report of a patient with limited-stage small cell lung cancer who presented with an HRR following concurrent chemoradiation therapy. Written informed consent was obtained from the patient.

\section{Case report}

In March 2013, a previously healthy 62-year-old male was referred to Inha University Hospital (Incheon, South Korea) due to small cell lung cancer diagnosed from a specimen obtained by bronchoscopic biopsy. The patient was a current smoker with no history of tuberculosis (TB). The Eastern Cooperative Oncology Group performance score (10) was zero. Smears and cultures for acid-fast bacilli were negative in a bronchial washing specimen. A mass on the lower lobe of the left lung and bulky lymphadenopathies on the left hilum, and mediastinal and supraclavicular areas, were noted on $\left[{ }^{18} \mathrm{~F}\right]$ fluorodeoxyglucose positron emission 
A

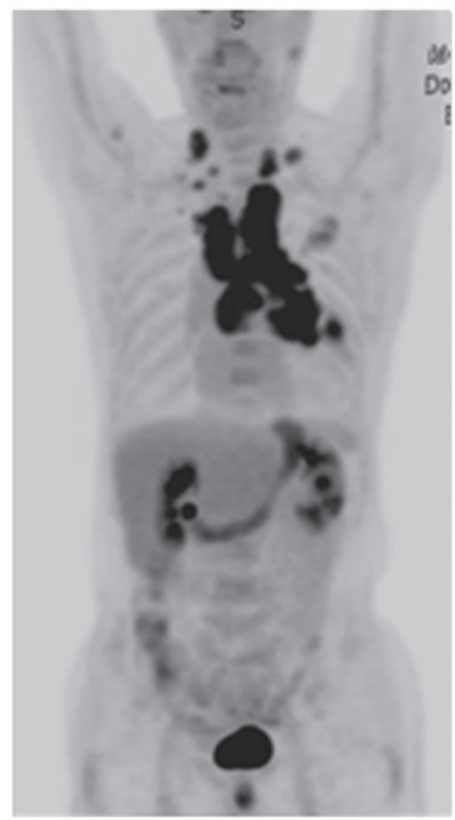

B

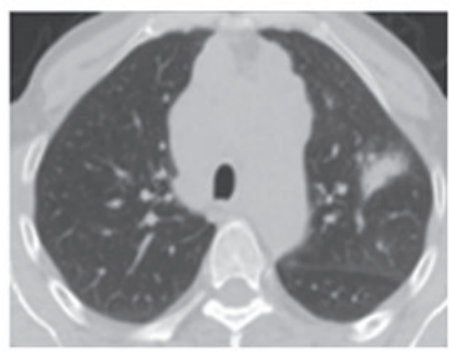

C

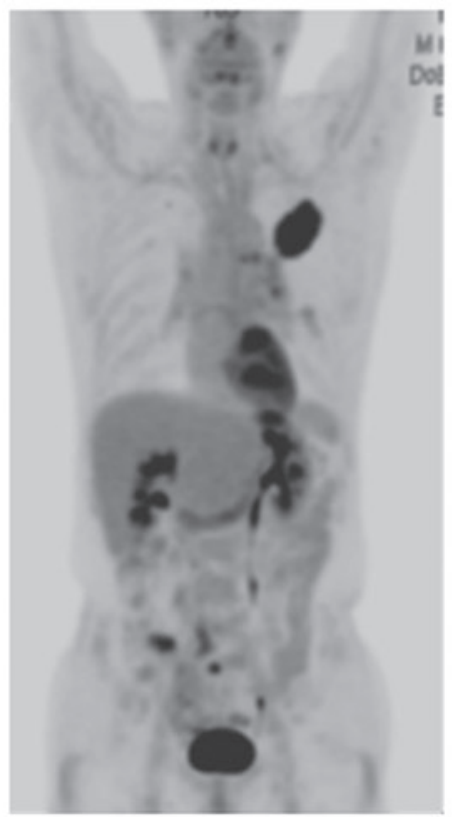

D

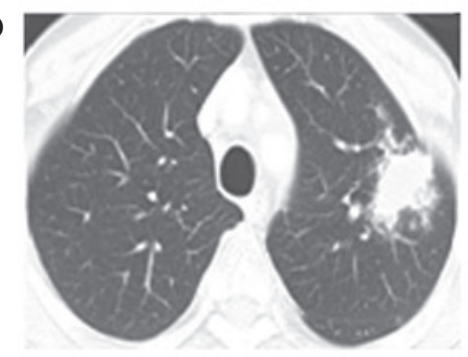

Figure 1. $\left[{ }^{18} \mathrm{~F}\right]$ fluorodeoxyglucose positron emission tomography/computed tomography scan (A and B) at the time of diagnosis and (C and D) after concurrent chemoradiation therapy.

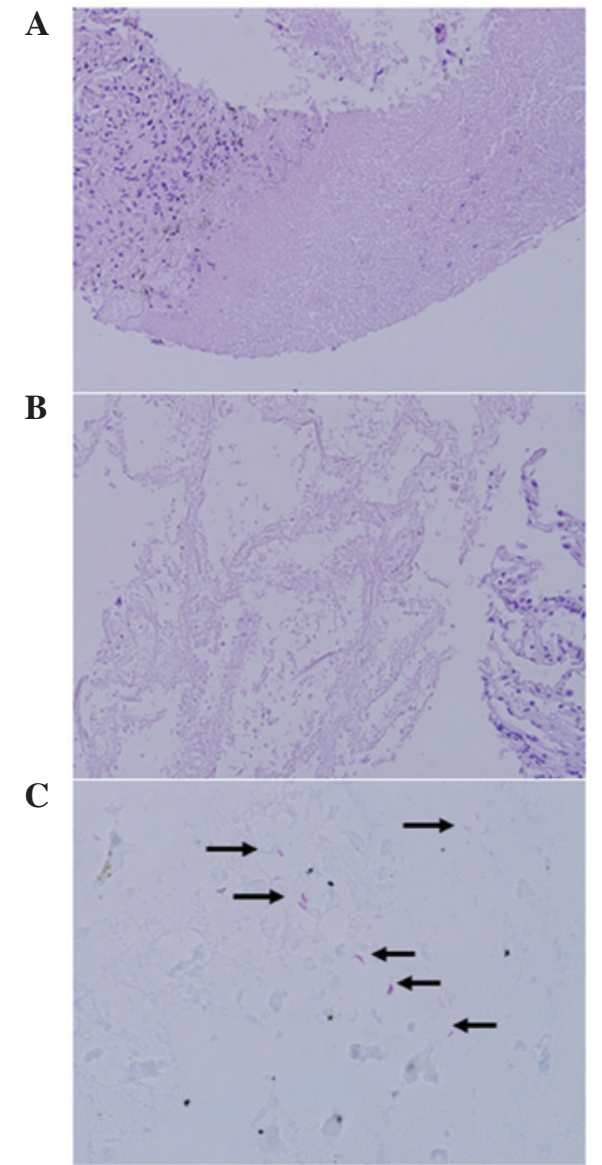

Figure 2. Histological features of CT-guided percutaneous needle biopsy for a lesion on the upper lobe of the left lung. (A) Nodular necrosis with fibrotic rim and (B) infarct-like necrosis of normal lung tissue were present without definite granuloma formation (hematoxylin and eosin stain; magnification, $\mathrm{x} 200$ ). (C) Several acid-fast bacilli were observed in the necrotic area (arrows) (Ziehl-Neelsen stain; magnification, x1,000).

tomography/computed tomography $\left({ }^{18}\right.$ FDG-PET-CT $)$ scans (Fig. 1A). A consolidative mass was observed on the upper lobe of the left lung (Fig. 1B). No evidence for metastasis was observed in other imaging studies, including bone scans and brain magnetic resonance imaging. Finally, the disease was classified as limited-stage disease, T4N3M0, using the seventh edition TNM staging system (11). Radiation therapy consisting of a total of 5,400 cGy was administered concurrently to the patient with chemotherapy for 1.5 months. All the aforementioned lesions were included in the radiation field. Chemotherapy consisted of etoposide $\left(100 \mathrm{mg} / \mathrm{m}^{2}\right.$ on days 1,2 and 3$)$ and cisplatin $\left(60 \mathrm{mg} / \mathrm{m}^{2}\right.$ on day 1$)$ every three weeks. Once the treatment was completed, response evaluation revealed that the lymphadenopathies and mass on the lower lobe of the left lung were apparently decreased in size (Fig. 1C), while the mass on the upper lobe of the left lung was significantly increased in size (Fig. 1D). The patient experienced no fever, coughing, sputum production or dyspnea. A blood examination showed a white blood cell count of $5.5 \times 10^{3} / \mathrm{ml}$ (normal range, $4-10 \times 10^{3} / \mathrm{ml}$ ), with $70 \%$ neutrophils and $17 \%$ lymphocytes, a C-reactive protein level of $1.18 \mathrm{mg} / \mathrm{dl}$ (normal range, $0-0.3 \mathrm{mg} / \mathrm{dl}$ ) and an erythrocyte sedimentation rate of $52 \mathrm{~mm} / \mathrm{h}$ (normal range for male individuals, $1-15 \mathrm{~mm} / \mathrm{h}$ ). A repeat bronchoscopic examination 
was performed for the evaluation of the aggravated lesion. No endobronchial lesions were found. Smears and polymeric chain reaction for acid-fast bacilli, and examinations for bacteria, fungi and malignancy were negative in a bronchial washing specimen. A CT-guided percutaneous needle biopsy was performed on the lesion. Pathological examination showed nodular necrosis with a fibrotic rim (Fig. 2A) and infarct-like necrosis of the normal lung tissue without definite granuloma formation (Fig. 2B). Several acid-fast bacilli in the necrotic area were identified on Ziehl-Neelsen stain (Fig. 2C). Next, anti-TB treatment [oral isoniazid (400 mg) rifampin $(600 \mathrm{mg})$ and ethambutol $(800 \mathrm{mg})$ plus pyrazinamide $(1,500 \mathrm{mg})]$ was administered to the patient. One month later, cultures for acid-fast bacilli were positive in a bronchial washing specimen. Drug susceptibility test results of Mycobacterium tuberculosis isolates showed sensitivity to all anti-TB drugs. The initial anti-TB regimen was administered for two months, and then isoniazid (400 mg), rifampin (600 mg) and ethambutol (800 mg) were administered in the following four months. Then, his tuberculous lesion was lessened and stabilized. His lung cancer was not recurred until April 2014 when he was lost to be followed up.

\section{Discussion}

The coincidence of active TB and lung cancer is an unusual clinical situation that is reported to occur in $0.3-0.6 \%$ of patients with lung cancer in TB endemic areas $(12,13)$. The question of whether chemotherapy or radiation therapy can reactivate latent $M$. tuberculosis infection in patients with lung cancer remains elusive (14). For instance, radiation causes a significant decrease in numbers of lymphocytes, which can led to the development of TB reactivation, while a clinical dose of radiation can directly kill $\sim 60 \%$ of $M$. tuberculosis $(15,16)$. Therefore, treatment can interact with TB in the fashion of a double-edged sword. No differences in clinical manifestation and outcome of anti-TB treatment were observed between patients with active TB occurring during chemotherapy in a previous study (17). With regard to the survival of patients with lung cancer, it has been reported that concomitant active TB can enhance local T-cell immunity and prolong survival (18). For these reasons, testing for latent TB is not recommended in these patients; clinicians are required to pay less attention to coincidence.

HRR is an existing concept that has witnessed a recent resurgence in use, as rapid advances in cancer genomics and functional imaging have facilitated our understanding of biological heterogeneity and its clinical application in the evaluation of treatment response. Consequently this can be of use in guiding further treatment and predicting survival in cancer patients $(6,7,9)$.

The TB diagnosis for the lesion on the upper lobe of the left lung in the current patient was so unlikely due to an absence of typical radiological patterns for TB on ${ }^{18} \mathrm{FDG}-\mathrm{PET}-\mathrm{CT}$ scan and negative results for acid-fast bacilli at the time of diagnosis. Therefore, the lesion was included in the radiation field during treatment on the assumption that this was malignant. In general, the response to concurrent chemoradiation therapy was expected to be higher, as this is reported to be $70-90 \%$ in limited-stage small cell lung cancer (19). Thus, it was not easy to predict the reason for this response when an HRR was obtained in the patient without histological confirmation.

In conclusion, when an HRR is observed, aggressive diagnostic approaches, including tissue biopsy for suspicious lesions, are useful in differentiating a non-tumor diagnosis from tumor heterogeneity and should be considered when the treatment provided is expected to have a higher response rate, particularly in TB endemic countries.

\section{Acknowledgements}

This study was supported by the National Research Foundation of Korea grant funded by the Korean government (Ministry of Science, ICT and Future Planning; no. NRF-2014R1A5A2009392), and the Basic Science Research Program through the National Research Foundation of Korea funded by the Ministry of Education, Science and Technology (no. 2013R1A1A2007537), and an Inha University Research Grant.

\section{References}

1. Vallières E, Shepherd FA, Crowley J, et al; International Association for the Study of Lung Cancer International Staging Committee and Participating Institutions: The IASLC Lung Cancer Staging Project: Proposals regarding the relevance of TNM in the pathologic staging of small cell lung cancer in the forthcoming (seventh) edition of the TNM classification for lung cancer. J Thorac Oncol 4: 1049-1059, 2009.

2. Rivera MP, Mehta AC and Wahidi MM: Establishing the diagnosis of lung cancer: Diagnosis and management of lung cancer, 3rd ed: American College of Chest Physicians evidence-based clinical practice guidelines. Chest 143 (Suppl 5): eS142-S65, 2013.

3. Noda K, Nishiwaki Y, Kawahara M, et al; Japan Clinical Oncology Group: Irinotecan plus cisplatin compared with etoposide plus cisplatin for extensive small-cell lung cancer. $\mathrm{N}$ Engl J Med 346: 85-91, 2002.

4. Chute JP, Chen T, Feigal E, Simon R and Johnson BE: Twenty years of phase III trials for patients with extensive-stage small-cell lung cancer: Perceptible progress. J Clin Oncol 17: 1794-1801, 1999.

5. Ross JS, Wang K, Elkadi OR, Tarasen A, Foulke L, Sheehan CE, Otto GA, Palmer G, Yelensky R, Lipson D, et al: Next-generation sequencing reveals frequent consistent genomic alterations in small cell undifferentiated lung cancer. J Clin Pathol 67: 772-776, 2014.

6. van Kessel CS, Samim M, Koopman M, van den Bosch MA Borel Rinkes IH, Punt CJ and van Hillegersberg R: Radiological heterogeneity in response to chemotherapy is associated with poor survival in patients with colorectal liver metastases. Eur J Cancer 49: 2486-2493, 2013.

7. Win T, Miles KA, Janes SM, Ganeshan B, Shastry M, Endozo R, Meagher M, Shortman RI, Wan S, Kayani I, et al: Tumor heterogeneity and permeability as measured on the CT component of PET/CT predict survival in patients with non-small cell lung cancer. Clin Cancer Res 19: 3591-3599, 2013.

8. Gerlinger M, Rowan AJ, Horswell S, et al: Intratumor heterogeneity and branched evolution revealed by multiregion sequencing. N Engl J Med 366: 883-892, 2012.

9. Chen ZY,Zhong WZ, Zhang XC, Su J, Yang XN, Chen ZH, Yang JJ, Zhou Q, Yan HH, An SJ, et al: EGFR mutation heterogeneity and the mixed response to EGFR tyrosine kinase inhibitors of lung adenocarcinomas. Oncologist 17: 978-985, 2012.

10. Oken MM, Creech RH, Tormey DC, et al: Toxicity and response criteria of the Eastern Cooperative Oncology Group. Am J Clin Oncol 5: 649-655, 1982.

11. Shepherd FA, Crowley J, Van Houtte P, Postmus PE, Carney D, Chansky K, Shaikh Z and Goldstraw P; International Association for the Study of Lung Cancer International Staging Committee and Participating Institutions: The International Association for the Study of Lung Cancer lung cancer staging project: Proposals regarding the clinical staging of small cell lung cancer in the forthcoming (seventh) edition of the tumor, node, metastasis classification for lung cancer. J Thorac Oncol 2: 1067-1077, 2007. 
12. Wu CY, Hu HY, Pu CY, Huang N, Shen HC, Li CP and Chou YJ Aerodigestive tract, lung and haematological cancers are risk factors for tuberculosis: An 8-year population-based study. Int J Tuberc Lung Dis 15: 125-130, 2011.

13. Kim HR, Hwang SS, Ro YK, Jeon CH, Ha DY, Park SJ, Lee CH Lee SM, Yoo CG, Kim YW, et al: Solid-organ malignancy as a risk factor for tuberculosis. Respirology 13: 413-419, 2008.

14. Nair R, Prabhash K, Sengar M, Bakshi A, Gujral S, Gupta S and Parikh P: The effect of short-term intensive chemotherapy on reactivation of tuberculosis. Ann Oncol 18: 1243-1245, 2007.

15. Stratton JA, Byfield PE, Byfield JE, Small RC, Benfield J and Pilch Y: A comparison of the acute effects of radiation therapy, including or excluding the thymus, on the lymphocyte subpopulations of cancer patients. J Clin Invest 56: 88-97, 1975.

16. Zack MB, Stottmeier K, Berg G and Kazemi H: The effect of radiation on microbiologic characteristics of $\mathrm{M}$ tuberculosis. Chest 66: 240-243, 1974.
17. Kim DK, Lee SW, Yoo CG, Kim YW, Han SK, Shim YS and Yim JJ: Clinical characteristics and treatment responses of tuberculosis in patients with malignancy receiving anticancer chemotherapy. Chest 128: 2218-2222, 2005.

18. Kuo CH, Lo CY, Chung FT, Lee KY, Lin SM, Wang CH, Heh CC, Chen HC and Kuo HP: Concomitant active tuberculosis prolongs survival in non-small cell lung cancer: A study in a tuberculosis-endemic country. PLoS One 7: e33226, 2012.

19. Edelman MJ, Chansky K, Gaspar LE, Leigh B, Weiss GR, Taylor SA, Crowley J, Livingston R and Gandara DR: Phase II trial of cisplatin/etoposide and concurrent radiotherapy followed by paclitaxel/carboplatin consolidation for limited small-cell lung cancer: Southwest oncology group 9713. J Clin Oncol 22: $127-132,2004$. 